A free and publicly available stimuli set for statistical learning experiments in language and music

Dominique T. Vuvan and Erica $\mathrm{H}$. Wojcik Skidmore College

February, 2020 


\begin{abstract}
The ability to encode regularities from the environment and abstract these patterns into structured cognitive representations is called statistical learning. Currently, one of the most studied paradigms of statistical learning is a word segmentation task originally introduced in a landmark paper by Saffran, Newport, and Aslin (1996). In this paradigm, participants hear a continuous stream of artificial words without acoustic cues to the boundaries separating the words. Then, they are asked to discriminate between words and non-words in the artificial language. One of the barriers that has impeded research progress using this paradigm is the lack of stimulus standardization. This lack of standardization is inefficient, causing researchers to create new stimuli for each new line of experiments. Furthermore, it makes the comparison of methods and results between different experiments difficult, if not impossible. The goal of the current report is to introduce a publically available stimuli set for language (syllable) and music (tone) segmentation experiments of statistical learning. All stimuli, data, and code associated with the current report are available for download on Open Science Framework, and we report here our validation experiments for both sets of stimuli.
\end{abstract}

Keywords: statistical learning, artificial language, syllables, tones, stimulus validity, replication 


\section{A free and publicly available stimuli set for statistical learning experiments in language and music}

In order to navigate their environment, humans must learn complex patterns present in sensory input. Over the past several decades, researchers have shown that infants, children, and adults are able to learn the structure of complex systems such as language and music by tracking statistical regularities (see Krogh, Vlach \& Johnson, 2013 for a review). Because this statistical learning mechanism has the potential to explain how humans learn and represent knowledge in multiple domains, there has been an explosion of research into its scope and constraints (Frost, Armstrong, \& Christiansen, 2019). In the most studied statistical learning paradigm, listeners are presented with a continuous stream of syllables and then asked to segment the stream into its constituent trisyllabic words (Saffran, Newport \& Aslin, 1996). By creating an artificial language, experimenters are able to manipulate the statistical regularities (typically transitional probabilities) between syllables while controlling all other properties of the input that might provide cues to structure. This allows researchers to attribute any observed segmentation to statistical learning, to the exclusion of other learning mechanisms or perceptual biases. This segmentation paradigm has been expanded to other domains, including musical tones (Saffran, Johnson, Aslin, \& Newport, 1999).

Despite the importance of statistical learning for theories of cognition, as well as the prevalence of the segmentation paradigm, researchers often create their own stimuli for each new line of research. This is not only inefficient, as researchers often recreate stimuli that already exist in other labs, but it also makes it difficult to compare statistical learning results across multiple experiments. For example, while the original studies of statistical learning in the language domain used computer generated monotone speech (Saffran, Newport \& Aslin, 1996), other replications and extensions have used a range of methods to create stimuli, such as recording and manipulating naturally produced syllables (Graf Estes et al., 2007) or using IBM's Watson (Hartshorne et al., 2019).

The decisions made by researchers when creating artificial language stimuli can be conceptualized as balancing the requirements of internal versus external validity. Researchers who prioritize internal validity will lean towards computer generation with strict standardization of parameters (e.g., Hartshorne et al., 2019). This ensures a high degree of control over the physical properties of stimuli. However, because this approach tends to ignore perceptual influences, stimuli produced in this manner may include unintended cues that come, for instance, from the perception of coarticulation between syllables. Further, the use of these stimuli may lead to global attentional or perceptual effects because they simply do not sound like natural speech, and thus may not be processed as such by listeners. For example, computer generated stimuli that are heavily controlled for amplitude and length may nevertheless include consonant onsets that are difficult to identify, or warped vowel sounds such that the intended syllable is not what is perceived.

On the other hand, researchers who prioritize external validity will lean towards the recording of natural speech, in order to reproduce a true-to-life listening experience for participants (e.g., Graf Estes et al., 2007; Pelucchi, Hay, \& Saffran, 2009). This approach ensures that participants will process the stimuli as speech, but has the major disadvantage of 
minimal control over the physical or perceptual properties of the stimuli. For instance, although Lew-Williams \& Saffran (2012) took measures to control for coarticulation cues, they did not report controlling other potential acoustic cues, such as length, amplitude, or harmonicity.

Most troubling, the majority of published reports on this word segmentation paradigm do not include a description of stimulus generation that is detailed enough for replication, nor do most research groups publicly share their stimuli for use across studies. In order to facilitate research on statistical learning and to encourage standardization of stimuli, this paper introduces a publically available stimuli set for language (syllable) and music (tone) segmentation experiments of statistical learning. All stimuli, data, and code associated with the current report are available on Open Science Framework at https://osf.io/9n6um/. For both domains, we tested the stimuli in two samples using a classic paradigm in which adult participants are exposed to 21 minutes of the language and then tested on their segmentation of the language with a two-alternative forced-choice (2AFC) task.

\section{Experiment 1: Syllable stimuli}

We exposed participants to syllable passages and used a 2AFC task to test whether they tracked the transitional probabilities between syllables. The experiment both served as a replication of Saffran, Newport \& Aslin (1996) and a confirmirmation of the generalization of the effect to our created syllable stimuli. We ran the experiment on Amazon's Mechanical Turk and with a university undergraduate population, both using Qualtrics software.

\section{Methods}

Participants. Based on the large effect sizes found by Saffran et al. (1996), we performed an a priori power analysis for a two-tailed single sample t-test; $n=20$ would give us $92 \%$ power. However, due to the small sample sizes of previous research, and thus the possibility of misestimated effect sizes, we aimed to test $n=40$. Our final sample size (see details below about exclusion criteria) was $n=41$ for the mTurk sample and $n=33$ for the undergraduate sample.

Stimuli. The stimuli were created to replicate those in Saffran et al. (1996) and consisted of three-syllable nonce words. However, unlike the original study, two languages were created to counterbalance for idiosyncrasies of the syllable sets. For Language 1, the words were babupu, bupada, dutaba, patubi, pidabu, tutibu. For Language 2, the words were tabupu, bupabi, pataba, titubi, pidata, tutipu. The words were created from single syllable recordings of a female native English speaker who was instructed to speak in a monotone voice (originally recorded for Wang \& Saffran, 2014). The syllables were then processed with Praat such that each syllable was acoustically monotone with the pitch normalized to $180 \mathrm{~Hz}$. The duration of each syllable was $330 \mathrm{~ms}$, and the intensity was normalized to $70 \mathrm{db}$. Each three-syllable nonce word was created by concatenating the three relevant syllables, such that each word was 990 ms long. Next, Matlab was used to randomly concatenate the six words of each language into three continuous streams of 1260 words (approximately seven minutes), containing no acoustic cues to word boundaries and no back-to-back repetitions of a particular word. This produced three unique training blocks and six individual words each for Language 1 and Language 2, 
which were all resampled to $16 \mathrm{kHz}$ and converted to $\mathrm{mp} 3$ format for the experiment. The between-words transitional probabilities were all 0 . Within the six words, the average transitional probabilities were $1.0,0.75,0.75,0.42,0.43$, and 0.5 for Language 1 and $1.0,0.5,0.5,0.5$, $0.45,0.42$, for Language 2.

Procedure. The procedure was identical to Saffran et al. (1996), except that participants controlled the length of breaks between the three training blocks; there were six catch trials at the end to check for participant attention; and the experiment was run online using Qualtrics and mTurk.

Training. After instructions to test sound volume, participants read the instructions, indicating that there were going to hear a continuous series of syllables in three seven-minute tracks. They were instructed to relax and avoid consciously analyzing the syllables, but to listen carefully because they would be tested on what they heard following the listening session. They were instructed to listen to each track without taking a break, but that they could take breaks between each track. After indicating that they were ready to begin, they were directed to a new page and the first seven-minute block began. Afterwards, a screen appeared asking the participant to click "next" when they were ready for the second track. The same procedure was then repeated for the third block. The order of the blocks were randomized for each participant.

Discrimination trials. After the last training block, participants read that they were going to hear 42 trials, each consisting of two words in succession. They were instructed to indicate which of the two words sounded most familiar. When the participant indicated they were ready, they first heard a practice trial consisting of two three-tone sequences. They were told that there was no right answer to the practice trial, and that it was just to demonstrate the format of the task.

After the practice trials, the 36 test trials began. Each trial consisted of a word from training and one of six part-words. Each part-word comprised two syllables from a word, as well as either a last or first tone that was rarely paired with the other two during training. Crucially, the languages were designed such that the words in Language 1 were the part words for Language 2, and vice versa. Thus, the discrimination trials were the same for participants in each Language condition, but the correct answer for each trial was reversed. Each word was tested against each part word once. The order of trials were randomized for each participant, and the order of the two tone words on each trial was counterbalanced across participants.

After the test trials, there were six catch trials to ensure that participants listened during the exposure phase. These trials pitted each of the six syllable words with a three-tone melody. Thus, to succeed on these trials, participants only needed to know that the exposure consisted of syllables, not tones. These trials immediately followed the test trials for all participants. The order of the stimuli within a trial and the order of the trials themselves was randomized across participants.

After the last trial, the participants were thanked and presented with a brief description of the experiment: "We are investigating how people learn patterns in language and music. The blocks of sounds that you heard in Part 1 consisted of 6 three-syllable 'words', and we are interested in the people's ability to identify those individual words." The entire experiment lasted approximately 30 minutes, depending on the length of breaks between training blocks. 


\section{Results - mTurk}

Participants were excluded if they did not reach $100 \%$ accuracy on the six catch trials ( $n$ $=15)$. Participants were also excluded if they did not finish the entire experiment $\left(n=114^{1}\right)$ or if at the end of the experiment, chose not to submit their results $(n=1)$. Following Saffran et al. (1996), we first compared performance across the two languages with a two-tailed independent sample t-test, finding that there was not a significant difference in accuracy $t(39)=0.72, p=$ $0.47,95 \% \mathrm{Cl}[-0.34,0.92]$. We also compared performance across whether participants used headphones or speakers with a two-tailed independent samples t-test, finding that there was not a significant difference in accuracy $t(39)=0.34, p=0.74,95 \% \mathrm{Cl}[-0.08,0.06]$. Thus, we collapsed across languages and audio presentation for all subsequent analyses.

To replicate the Saffran et al. (1996) analysis, we used a two-tailed single sample t-test to compare performance to chance. Adults performed significantly above chance, $t(40)=3.83, p$ $<0.001,95 \% \mathrm{Cl}[0.53,0.60], d=0.55(M=0.56, S D=0.11)$. We then averaged the two transitional probabilities in the three-syllable words and split the six test items into three high and three low TP words for Language $1^{2}$. High TPs were 1, 0.75, and 0.75 and low TPs were $0.42,0.43$, and 0.5. A two-tailed paired t-test was used to compare high and low TP performance, finding that performance was not significantly better for the high TP words, $t(20)=$ $0.04, p=0.97,95 \% \mathrm{Cl}[-0.07,0.07]$. We also ran a two-tailed correlation between TP and performance for the items, finding that there was not a significant correlation, $r(10)=0.22, p=$ $0.49,95 \% \mathrm{Cl}[-0.71,0.40]$. These item analyses are underpowered and thus the null results should be interpreted with caution.

To test whether the effects generalize beyond the stimulus items, and to account for the fact the accuracy DV is not a continuous measure, we also analysed the data with a binomial mixed effects model (based on a recent replication of Saffran et al., 1996 by Hartshorne et al., 2019). Subject and item were random effects. The model estimated separate intercepts and slopes for each item. The intercept was not significantly greater than chance (Wald's $z=1.65, p$ $=0.10)$.

\section{Results - Undergraduate Sample}

Participants were excluded if they did not reach $100 \%$ accuracy on the six catch trials ( $n$ = 29). Participants were also excluded if they did not finish the entire experiment $(n=29)$.

Following Saffran et al. (1996), we first compared performance across the two languages with a two-tailed independent samples t-test, finding that there was not a significant difference in accuracy $t(31)=-0.13, p=0.89,95 \% C l[-0.05,0.05]$. We also compared performance across whether participants used headphones or speakers (one participant did not answer this question) with a two-tailed independent samples t-test, finding that there was not a significant

\footnotetext{
${ }^{1}$ While we did not expect the number of dropouts, we suspect that it is due to the length of the exposure blocks and repetitiveness of the study.

${ }^{2}$ We replicated the High vs. Low TP analysis in Saffran et al. (1996) only for Language 1 because Language 2 (based on Experiment 2 in Saffran et al., 1996) did not have a high-low transitional probability split: three of the tone words had an average TP of 0.5 , one had an average TP of 0.42 , one had an average TP of 0.45 , and the last had an average TP of 1.0 .
} 
difference in accuracy $t(30)=0.36, p=0.72,95 \% \mathrm{Cl}[-0.04,0.05]$. Thus, we collapsed across languages and audio presentation for all subsequent analyses.

To replicate the Saffran et al. (1996) analysis, we used a two-tailed single sample t-test to compare performance to chance. Adults did not perform significantly above chance, $t(32)=$ $0.87, p=0.39,95 \% C l[0.48,0.53], d=0.14(M=0.51, S D=0.07)$. We then averaged the two transition probabilities in the three-tone words and split the six test items into three high and three low TP words for Language 1. A two-tailed paired t-test was used to compare high and low TP performance, finding that performance was not significantly better for the high TP words, $t(15)=0.90, p=0.38,95 \% \mathrm{Cl}[-0.06,0.15]$. We also ran a two-tailed correlation between TP and performance for the items, finding that there was a significant correlation, $r(10)=0.14, p=$ $0.66,95 \% \mathrm{Cl}[-0.47,0.66]$. These analyses are underpowered and thus the null results should be interpreted with caution.

To test whether the effects generalize beyond the stimulus items, and to account for the fact the accuracy DV is not a continuous measure, we also analysed the data with a binomial mixed effects model (based on a recent replication of Saffran et al., 1996 by Hartshorne et al. 2019). Subject and item were random effects. The model estimated separate intercepts and slopes for each item. The intercept was not significantly greater than chance (Wald's $z=0.43, p$ $=0.67$ ).

\section{Experiment 2: Tone stimuli}

We exposed participants to tone passages and used a 2AFC task to test whether they tracked the transitional probabilities between tones. The experiment both served as a replication of Saffran et al. (1999) and to confirm generalization of the effect to our created tone stimuli. We ran the experiment on Amazon's Mechanical Turk and with a university undergraduate population using Qualtrics software.

\section{Methods}

Participants. Based on the large effect sizes found by Saffran et al. (1999), we performed an a priori power analysis for a two-tailed single sample t-test; $n=20$ would give us $92 \%$ power. However, due to the small sample sizes of previous research, and thus the possibility of misestimated effect sizes, we aimed to test $n=40$. However, due to our exclusion criteria (see details below), our final samples were $n=37$ (mTurk) and $n=25$ (undergraduate sample).

Stimuli. As in Saffran et al. (1999), the stimuli consisted of three-tone words. To counterbalance for idiosyncrasies of the tones, two sets of stimuli were made, each consisting of six three-tone words. For Language 1, the tone words were ADB, CC\#D, D\#ED, DFE, FCF\#, GG\#A. For Language 2, the tone words were C\#CF\#, CC\#B, D\#EG\#, DFF\#, FG\#A, G\#DB. These tone words were synthesized as wav files in Audacity. Individual pure tones were $330 \mathrm{~ms}$ long and produced at a sampling rate of $8 \mathrm{kHz}$. Each tone word was therefore $990 \mathrm{~ms}$ long.

Next, Matlab was used to randomly concatenate the six tone words of each language into three continuous streams of 1260 tone words (approximately seven minutes), containing no acoustic cues to word boundaries and no back-to-back repetitions of a particular tone word. This 
produced three unique training tracks and six individual tone words each for Language 1 and Language 2, which were all converted to $\mathrm{mp} 3$ format for the experiment. Because we used the same structure, the transitional probabilities were the same as the language stimuli (see Experiment 1).

Procedure. The procedure was identical to Experiment 1, except that tone words were used instead of syllable words. As in Experiment 1, the test trials were the same for each language condition due to the fact that the tone words in Language 1 were the part tone words for Language 2 and vice versa. The six catch trials were identical to Experiment 1, but the correct responses were the tone words, not the syllable words.

\section{Results - MTurk sample}

Participants were excluded if they did not reach $100 \%$ accuracy on the six catch trials ( $n$ = 23). Participants were also excluded if they did not finish the entire experiment or if at the end of the experiment, chose not to submit their results $(n=80)$. Following Saffran et al. (1999), we first compared performance across the two languages with a two-tailed independent samples t-test, finding that there was not a significant difference in accuracy $t(35)=1.53, p=0.14,95 \%$ $\mathrm{Cl}[-0.02,0.17]$. We also compared performance across whether participants used headphones or speakers with a two-tailed independent samples t-test, finding that there was not a significant difference in accuracy $t(35)=0.97, p=0.34,95 \% \mathrm{Cl}[-0.06,0.15]$. Thus, we collapsed across languages and audio presentation for all subsequent analyses.

To replicate the Saffran et al. (1999) analysis, we used a two-tailed single sample t-test to compare performance to chance. Adults performed significantly above chance, $t(36)=2.59, p$ $=0.01,95 \% \mathrm{Cl}[0.51,0.61], d=0.4(M=0.56, S D=0.15)$. We then averaged the two transition probabilities in the three-tone words and split the six test items into three high and three low TP words for Language 1. High TPs were 1, 0.75, and 0.75 and low TPs were $0.42,0.43$, and 0.5 (see Experiment 1 for details further details). A two-tailed paired t-test was used to compare high and low TP performance, finding that performance was not significantly better for the high TP words, $t(18)=1.41, p=0.18,95 \% \mathrm{Cl}[-0.19,0.04]$. We also ran a two-tailed correlation between TP and performance for the items across both languages, finding that there was not a significant correlation, $r(10)=0.08, p=0.81,95 \% \mathrm{Cl}[-0.52,0.63]$. These analyses are underpowered and the null results should thus be interpreted with caution.

To test whether the effects generalize beyond the stimulus items, and to account for the fact the accuracy DV is not a continuous measure, we also analysed the data with a binomial mixed effects model (based on a recent replication of Saffran et al., 1996 by Hartshorne et al. 2019). Subject and item were random effects. The model estimated separate intercepts and slopes for each item. The intercept was significantly greater than chance (Wald's $z=2.07, p=$ 0.04).

\section{Results - Undergraduate sample}

Participants were excluded if they did not reach $100 \%$ accuracy on the six catch trials ( $n$ $=56)$. Participants were also excluded if they did not finish the entire experiment $(n=31)$ or if at the end of the experiment, chose not to submit their results $(n=4)$. Following Saffran et al. (1999), we first compared performance across the two languages with a two-tailed independent 
samples t-test, finding that there was not a significant difference in accuracy $t(23)=0.26, p=$ $0.79,95 \% \mathrm{Cl}[-0.08,0.06]$. We also compared performance across whether participants used headphones or speakers (one participant was excluded from this analysis only for not providing an answer to this question) with a two-tailed independent samples t-test, finding that there was not a significant difference in accuracy $t(22)=0.29, p=0.78,95 \% \mathrm{Cl}[-0.09,0.06]$. Thus, we collapsed across languages and audio presentation for all subsequent analyses.

To replicate the Saffran et al. (1999) analysis, we used a two-tailed single sample t-test to compare performance to chance. Adults performed significantly above chance, $t(24)=4.10, p$ $<0.001, d=0.88(M=0.57, S D=0.08)$. We then averaged the two transition probabilities in the three-tone words and split the six test items into three high and three low TP words for Language 1. High TPs were $1,0.75$, and 0.75 and low TPs were $0.42,0.43$, and 0.5. A two-tailed paired t-test was used to compare high and low TP performance, finding that performance was not significantly better for the high TP words, $t(11)=0.06, p=0.95,95 \% \mathrm{Cl}$ $[-0.12,0.11]$. We also ran a two-tailed correlation between TP and performance for the items across both languages, finding that there was not a significant correlation, $r(10)=0.13, p=0.69$, $95 \% \mathrm{Cl}[-0.48,0.66]$. These analyses are underpowered and the null results should thus be interpreted with caution.

To test whether the effects generalize beyond the stimulus items, and to account for the fact the accuracy DV is not a continuous measure, we also analysed the data with a binomial mixed effects model (based on a recent replication of Saffran et al., 1996 by Hartshorne et al. 2019). Subject and item were random effects. The model estimated separate intercepts and slopes for each item. The intercept was significantly greater than chance (Wald's $z=2.27, p=$ 0.02).

\section{Discussion}

The goal of the current experiments was to create and share publically a set of stimuli for use in word segmentation experiments of statistical learning. More specifically, based on experiments by Saffran et al. (1996; 1999), we set out to create artificial languages of both syllables and tones such that the only cue to word boundaries were the transitional probabilities between words. While this type of artificial language has been used in many experiments across the past decades (see Frost et al., 2019 for a review), the stimuli vary in how they are created and processed (and reported), and most stimuli sets are not shared publicly.

We created two artificial language structures of three-syllable words based on the original Saffran et al (1996) statistical learning experiment. Three seven-minute exposure blocks were created for each language such that the transitional probabilities between the words were low and the transitional probabilities within the word syllables was high; there were no other cues to word boundaries (such as pauses, amplitude or pitch changes, or coarticulation information). We then created 72 two-alternative forced-choice test trials for each language. Each trial comprised a three-syllable word and a three-syllable part word. The part word consisted of two syllables from a word in the language, plus a different final syllable. We generated the artificial languages for two domains: language and music. In the language domain, each syllable was a consonant and vowel from the American English phoneme 
inventory. In the music domain, each syllable was a pure tone. Because the structure of the languages remain the same across domains, their learnability can be directly compared.

To verify that our stimuli were segmentable, we replicated the Saffran et al. 1996 and 1999 experiments. We exposed participants to either the music or language blocks, then tested whether they were able to identify the three-syllable words as familiar. Across two different samples for each domain and across both artificial language structures, we generally replicated the original effects. Paired samples t-tests revealed that participants selected the three-word syllables from the language at levels significantly above chance in both samples in the music domain and one sample in the language domain, with no differences across the two different language structures. Thus, we found evidence that when exposed to our stimuli, participants were able to use the transitional probabilities in the languages to segment the stream into three-unit chunks, both in the language and music conditions.

We also ran binomial mixed-effects models to address the non-scalar aspect of the dependent variable, as well as take into account participant and item variability (following Hartshorne et al., 2019). Although this analysis confirmed segmentation in the music domain, it did not confirm segmentation in the language domain. The inconsistency across analyses is addressed further below.

\section{Limitations}

We discuss the limitations of our stimulus set separately for the language and tone domains. For the language domain, the main limitations are external validity and generalizability. Our stimuli are created from consonants and vowels from the American English phoneme inventory. Thus, they are most appropriate for use with native speakers of American English. We also did not find evidence for segmentation with a binomial mixed-effects model, and the paired t-test was not significant in one of our samples. Notably, Hartshorne et al. (2019) also did not find a significant effect with a binomial mixed-effects model in their replication of statistical learning in the language domain (with a different stimuli set). This suggests that the segmentation effect, though present with the current stimulus set, may not generalize to different items and people. This is perhaps not surprising because of the difficulty in balancing external validity and stimulus control in language stimuli (as discussed in the Introduction). Because our stimuli were made from naturally produced speech, it is possible that there are unidentified features of how the syllables are perceived that either facilitate or inhibit segmentation. We did not want to sacrifice external validity in our experiments and so must accept the lack of complete control. Overall, our analyses suggest that our language stimuli are appropriate for many tests of statistical learning. Researchers, however, should continue to explore the perceptual biases in and limits of artificial word segmentation stimuli.

For the music domain stimuli, the main limitation is external validity. While pure tones are often used in studies of statistical learning in the music domain (Herff et al., 2017; Vuvan, 2012), it is unclear if they are perceived as music. Repeated sounds are often interpreted as music-like (Margulis \& Simchy-Gross, 2016; Simchy-Gross \& Margulis, 2018), but the pure tone blocks in the current experiments initially sound robotic. However, by using pure tones, the stimuli can be maximally controlled for amplitude, frequency, pitch and length. Notably, because pure tones consist of a simple sinusoidal waveform, they are easier to control than a complex harmonic 
sound like a syllable. Thus, the tones are by definition a more controlled set of stimuli than the syllables. Indeed, this may account for why the mixed-effects models showed a significant segmentation effect for the tones only. Participants may have had fewer perceptual cues influencing their behavior for the tone stimuli, making the statistical learning signal clearer. Thus, while the tone stimuli are perhaps appropriate for a wider range of statistical learning experiments because they are not specific to a certain culture and do not include potentially unidentified perceptual biases, they are limited by the lack of external validity. Again, as with the language stimuli, researchers must continue to consider and experimentally investigate the balance of external and internal validity.

\section{Conclusion}

Cognitive scientists continue to frequently use word segmentation experiments to study statistical learning, a potentially powerful mechanism behind how humans find structure in the world. The lack of standardization, transparency, and public sharing of stimuli is not only inefficient, but also makes it difficult to compare and synthesize results across studies. We encourage researchers to freely use our stimuli and to reach out to us if they have any questions. 


\section{References}

Frost, R., Armstrong, B. C., \& Christiansen, M. H. (2019). Statistical learning research: A critical review and possible new directions. Psychological Bulletin, 145(12), 1128.

Graf Estes, K., Evans, J. L., Alibali, M. W., \& Saffran, J. R. (2007). Can infants map meaning to newly segmented words? Statistical segmentation and word learning. Psychological Science, 18(3), 254-260.

Hartshorne, J. K., Skorb, L., Dietz, S. L., Garcia, C. R., lozzo, G. L., Lamirato, K. E., ... Vidal, J. (2019). The Meta-Science of Adult Statistical Word Segmentation: Part 1. Collabra: Psychology, $5(1), 1$.

Herff, S. A., Olsen, K. N., Dean, R. T., \& Prince, J. (2018). Memory for melodies in unfamiliar tuning systems: Investigating effects of recency and number of intervening items. Quarterly Journal of Experimental Psychology, 71(6), 1367-1381.

Krogh, L., Vlach, H., \& Johnson, S. P. (2013). Statistical learning across development: Flexible yet constrained. Frontiers in Psychology, 3, 598.

Lew-Williams, C., \& Saffran, J. R. (2012). All words are not created equal: Expectations about word length guide infant statistical learning. Cognition, 122(2), 241-246.

Margulis, E. H., \& Simchy-Gross, R. (2016). Repetition enhances the musicality of randomly generated tone sequences. Music Perception: An Interdisciplinary Journal, 33(4), 509-514.

Pelucchi, B., Hay, J. F., \& Saffran, J. R. (2009). Learning in reverse: Eight-month-old infants track backward transitional probabilities. Cognition, 113(2), 244-247.

Saffran, J. R., Newport, E. L., \& Aslin, R. N. (1996). Word segmentation: The role of distributional cues. Journal of Memory and Language, 35(4), 606-621.

Saffran, J. R., Johnson, E. K., Aslin, R. N., \& Newport, E. L. (1999). Statistical learning of tone sequences by human infants and adults. Cognition, 70(1), 27-52.

Simchy-Gross, R., \& Margulis, E. H. (2018). The sound-to-music illusion: Repetition can musicalize nonspeech sounds. Music \& Science, 1, 2059204317731992.

Vuvan, D. (2012). The Statistical Learning Of Musical Expectancy. University of Toronto (Canada).

Wang, T., \& Saffran, J. R. (2014). Statistical learning of a tonal language: The influence of bilingualism and previous linguistic experience. Frontiers in Psychology, 5, 953. 